\title{
The Ablation-Front Rayleigh-Taylor Dispersion Curve in Indirect Drive
}

K. S. Budil, B. Lasinski, M. J. Edwards, A. S. Wan, B. A. Remington, S. V. Weber, S. G. Glendinning, L. S. Suter, and P. Stry

This article was submitted $42^{\text {nd }}$ Annual Meeting of the APS Division of Plasma Physics, Quebec City, Canada, October 23 - 27, 2000

\section{November 17, 2000}

Lawrence

Lutromore

National.

Laboratory 


\section{DISCLAIMER}

This document was prepared as an account of work sponsored by an agency of the United States Government. Neither the United States Government nor the University of California nor any of their employees, makes any warranty, express or implied, or assumes any legal liability or responsibility for the accuracy, completeness, or usefulness of any information, apparatus, product, or process disclosed, or represents that its use would not infringe privately owned rights. Reference herein to any specific commercial product, process, or service by trade name, trademark, manufacturer, or otherwise, does not necessarily constitute or imply its endorsement, recommendation, or favoring by the United States Government or the University of California. The views and opinions of authors expressed herein do not necessarily state or reflect those of the United States Government or the University of California, and shall not be used for advertising or product endorsement purposes.

This is a preprint of a paper intended for publication in a journal or proceedings. Since changes may be made before publication, this preprint is made available with the understanding that it will not be cited or reproduced without the permission of the author.

This work was performed under the auspices of the United States Department of Energy by the University of California, Lawrence Livermore National Laboratory under contract No. W-7405-Eng-48.

This report has been reproduced directly from the best available copy.

Available electronically at http://www.doc.gov/bridge

Available for a processing fee to U.S. Department of Energy

And its contractors in paper from

U.S. Department of Energy

Office of Scientific and Technical Information

P.O. Box 62

Oak Ridge, TN 37831-0062

Telephone: (865) 576-8401

Facsimile: (865) 576-5728

E-mail: reports@adonis.osti.gov

Available for the sale to the public from

U.S. Department of Commerce

National Technical Information Service

5285 Port Royal Road

Springfield, VA 22161

Telephone: (800) 553-6847

Facsimile: (703) 605-6900

E-mail: orders@ntis.fedworld.gov

Online ordering: http://www.ntis.gov/ordering.htm

OR

Lawrence Livermore National Laboratory

Technical Information Department's Digital Library

http://www.llnl.gov/tid/Library.html 


\title{
The ablation-front Rayleigh-Taylor dispersion curve in indirect drive
}

\author{
K. S. Budil, B. Lasinski, M. J. Edwards, A. S. Wan, B. A. Remington, S. V. Weber, S. G. \\ Glendinning, L. Suter, and P. E. Stry \\ Lawrence Livermore National Laboratory \\ P. O. Box 808, L-21, Livermore, California 94550
}

(November 17, 2000)

\begin{abstract}
The Rayleigh-Taylor (RT) instability, which occurs when a lower-density fluid accelerates a higher-density layer, is common in nature. At an ablation front a sharp reduction in the growth rate of the instability at short wavelengths can occur, in marked contrast to the classical case where growth rates are highest at the shortest wavelengths. Theoretical and numerical investigations of the ablative RT instability are numerous and differ considerably on the level of stabilization expected. We present here the results of a series of laser experiments designed to probe the roll-over and cutoff region of the ablation-front RT dispersion curve in indirect drive. Aluminum foils with imposed sinusoidal perturbations ranging in wavelength from 10 to $70 \mu \mathrm{m}$ were ablatively accelerated with a radiation drive generated in a gold cylindrical hohlraum. A strong shock wave compresses the package followed by an $\sim 2$ ns period of roughly constant acceleration and the experiment is diagnosed via face-on radiography. Perturbations with wavelengths $\geq 20 \mu \mathrm{m}$ experienced substantial growth during the acceleration phase while shorter wavelengths showed a sharp drop off in overall growth. These experimental results compared favorably to calculations with a 2-D radiation-hydrodynamics code,
\end{abstract}


however, the growth is significantly affected by the rippled shock launched by the drive. We performed numerical simulations to elucidate the influence of the rippled shock wave on the eventual growth of the perturbations, allowing comparisons to the analytic model developed by Betti et al. This combination of experiments, simulations and analytic modeling illustrates the qualitative simplicity yet quantitative complexity of the compressible RT instability. We have measured the Rayleigh-Taylor (RT) dispersion curve for a radiativelydriven sample in a series of experiments on the Nova laser facility. Planar aluminum foils were ablatively-accelerated and the subsequent perturbation growth was diagnosed via $\mathrm{x}$-ray radiography. These measurements unambiguously map out the linear regime dispersion curve, including the observation of stabilization at short wavelengths. The data are compared favorably to twodimensional simulations. Due to the influence of the rippled shock transit phase of the experiment, direct comparison to the ablation front RT theory of R. Betti was difficult. Instead, a numerical "experiment" was constructed that minimized the influence of the shock and this was compared to the Betti model showing quite good agreement. This work was performed under the auspices of the U.S. Department of Energy by the University of California, Lawrence Livermore National Laboratory under contract No. W-7405-Eng48. 
The interface between the two fluids of different densities is unstable via the RayleighTaylor instability whenever a lower-density fluid accelerates a higher-density layer. [1] Examples of this buoyancy-driven instability are numerous in nature, ranging from imploding inertial confinement fusion capsules to $Z$ pinches to exploding supernovae. It is manifested as broad bubbles of the lower density material rising up past falling spikes of the high density material. At an ablation front a sharp reduction in the growth rate of the instability at short wavelengths can occur, in marked contrast to the classical case where growth rates are highest at the shortest wavelengths. The outer surface of an ICF capsule is an example of the ablation front instability, where the acceleration of the shell is provided by the lowdensity, ablated plasma. A second, potential astrophysical occurrence of ablation front RT, a photoevaporation front, may be the mechanism forming the beautiful "pillars of creation" in the Eagle nebula [2].

Much of the research on the RT instability in recent years has been motivated by the inertial confinement fusion (ICF) community since in both the direct- and indirect-drive schemes for ICF, the imploding capsule experiences two phases of RT instability. [3,4] The ablation front is unstable during the acceleration phase and the pusher-fuel interface becomes unstable during deceleration and stagnation. Strong perturbation growth can lead to spikes of the capsule wall material protruding into the fuel, which can severely degrade capsule performance. [5] In indirect-drive, where the driver energy is converted into x-rays in a high$\mathrm{Z}$ cavity (hohlraum) which irradiate the capsule, the ablation velocities $\mathrm{v}_{\mathrm{a}}=(\mathrm{dm} / \mathrm{dt}) / \rho_{\max }$, where $\mathrm{m}$ is the foil mass per unit area and $\rho_{\max }$ is the maximum density at the ablation front, are high and short wavelength modes are predicted to be stabilized. However, for direct-drive the ablation velocities are lower and the stabilizing effect is expected to occur at shorter wavelengths. Despite considerable effort worldwide, there has been no conclusive measurement of the RT cutoff wavelength at an ablation front in indirect drive. For direct drive the cutoff wavelengths are expected to be below standard diagnostic resolution limits [6] of $10 \mu \mathrm{m}$ and prior indirect drive measurements utilized a rapidly varying drive, making a dispersion curve assessment difficult. $[7,8]$ We present here the first conclusive experimental 
measurement of the ablation front RT cutoff utilizing a nearly steady-state $\mathrm{x}$-ray drive to accelerate a rippled, aluminum foil.

Theoretical and numerical investigations of the ablative RT instability are numerous and differ considerably on the level of stabilization expected. $[3-5,9,7,10]$ We present here a comparison of the theoretical model for ablation front RT proposed by Betti et al. with experimental data from an indirect drive geometry. [11] The data are well-reproduced by two-dimensional simulations. The first-principles Betti model was seen to qualitatively reproduce the measured dispersion curve for aluminum however, a more detailed, quantitative comparison was complicated due to the presence of a strong, rippled shock in the experiment that was not accounted for in the model. Therefore a numerical "experiment" was conducted that minimized the influence of shock waves to facilitate the comparison to the model. This comparison showed quite good, quantitative agreement between the simulation and model. As expected, the standard Takabe formulation applied with $\beta$ used as a fitting parameter provided excellent agreement with the numerical experiment.

The experimental configuration is illustrated in Figure 1. A 750- $\mu \mathrm{m}$-diameter, 25- $\mu \mathrm{m}$ thick planar, aluminum foil is mounted across a hole on a 4 -mm-long by 2.32 -mm-diameter cylindrical gold hohlraum.
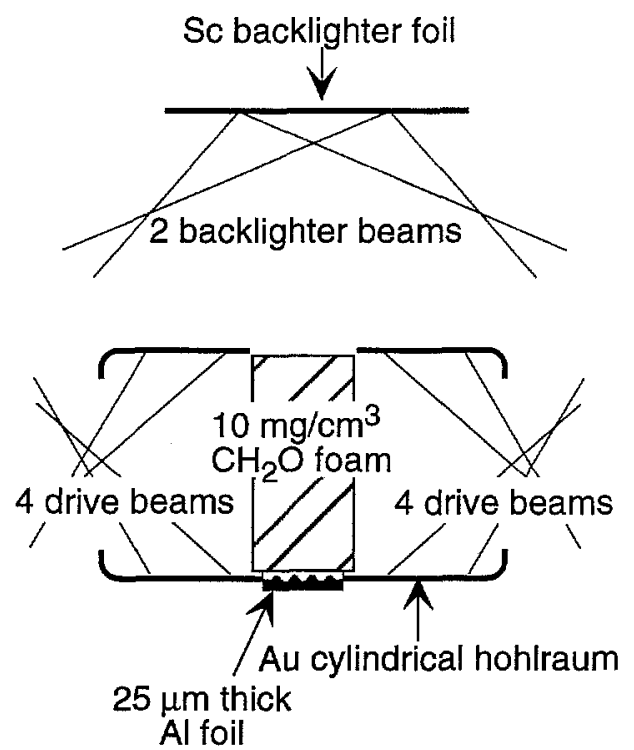

FIG. 1. Experimental schematic for the ablation front RT measurements in aluminum. 
The central $1 \mathrm{~mm}$ of the hohlraum is filled with $10 \mathrm{mg} / \mathrm{cm}^{3} \mathrm{CH}_{2} \mathrm{O}$ (Agar) foam to keep the diagnostic line-of-sight from filling with high-opacity gold from the hohlraum wall for the duration of the measurement. Calculations have been done to confirm that this low-density foam does not have a significant effect on the hydrodynamic evolution of the aluminum foil. Eight of the ten Nova laser beams at $0.35 \mu \mathrm{m}$ wavelength are focussed into the hohlraum and generate $\mathrm{a} \sim 100 \mathrm{eV}$ radiation drive. The pulse is carefully temporally shaped, with an overall $\sim 6$ ns duration, to produce a nearly constant acceleration over a period of approximately 2 ns. The pulse shape and radiation drive are shown in Figure 2(a). Two 2 ns square pulses at $0.35 \mu \mathrm{m}$ wavelength are delayed relative to the drive beams and focussed onto a scandium backlighter foil. This generates a hard $\mathrm{x}$-ray spectrum dominated by the $4.3 \mathrm{keV}$ He- $\alpha$ line which back-illuminates the accelerating, planar foil. A gated x-ray pinhole camera is used to record a sequence of two-dimensional x-ray images. [12] The perturbation amplitude as a function of time is proportional to the changing optical depth (OD) modulation in the aluminum foil. 

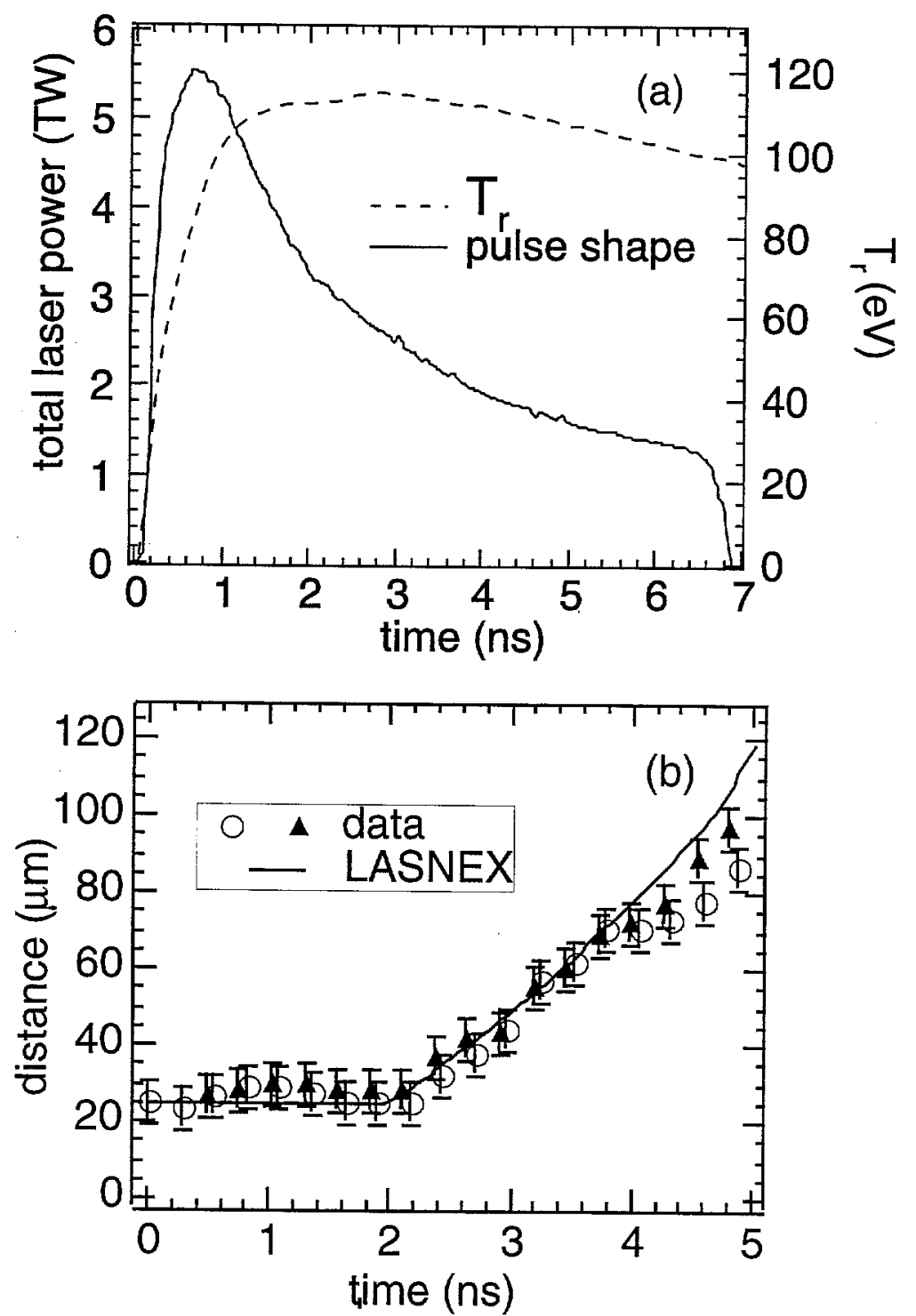

FIG. 2. (a) Total laser power profile utilized in the aluminum experiments and the corresponding hohlraum radiation temperature profile simulated using this laser pulse shown as a function of time. (b) Rear surface position as a function of time from the simulations (solid line) and experimental measurement (points).

The radiation drive used in the simulations was designed via a two-dimensional LASNEX [13] hohlraum simulation. The calculated drive profile was verified by several experimental measurements. These included a side-on foil trajectory measurement as well as shock breakout measurements through a stepped aluminum witness foil placed on the side of the hohlraum, both measures of the gross hydrodynamics. The data were well-reproduced by 
one-dimensional simulations utilizing the calculated $\mathrm{T}_{\mathrm{r}}(\mathrm{t})$ profile. Figure $2(\mathrm{~b})$ shows the measured and calculated side-on foil trajectory, which indicates that the foil does not accelerate as a unit until the initial shock breaks out of the rear surface of the $\mathrm{Al}$ at $\mathrm{t} \sim 2 \mathrm{~ns}$. The experimental measurements of perturbation growth were taken after shock breakout to focus on the RT evolution of the foil rather than the dynamics of the shock passage phase. However, because the growth is measured in transmission as $\Delta(O D)$ any modulations in the foil away from the ablation front due to the passage of the shock will be visible in the data and its influence must be considered.

The planar Al foils used in the experimental measurements were $25 \mu \mathrm{m}$ thick and had a variety of sinusoidal modulations machined onto one side, which was then placed facing the x-ray drive. Except for the longest wavelength investigated, $\lambda=70 \mu \mathrm{m}$, each target had side-by-side patterns of sinusoidal ripples separated by a small gap so that the small variations in the x-ray drive for each measurement could be accounted for in the calculations. The pairs of wavelength groups investigated were $\lambda=10 \mu \mathrm{m}$ with $\lambda=30 \mu \mathrm{m}, \lambda=20 \mu \mathrm{m}$ with $\lambda=50 \mu \mathrm{m}$, and $\lambda=12 \mu \mathrm{m}$ with $\lambda=16 \mu \mathrm{m}$ and $\lambda=20 \mu \mathrm{m}$. Wavelengths of 20,30 , 50 and $70 \mu \mathrm{m}$ were given an initial amplitude $\eta_{o}=1.0 \mu \mathrm{m}$ while $\lambda=10,12$, and $16 \mu \mathrm{m}$ had $\eta_{0}=0.5 \mu \mathrm{m}$. The exact shape of the perturbations was characterized by atomic force microscopy and contact profilometry and is indeed a single-mode sinusoid, known to better than $10 \%$.

Typical images from the experimental data are shown in Figure 3 along with the corresponding averaged lineouts displayed in $\ln ($ exposure $) \propto \rho \kappa \mathrm{dz} \sim \delta(\mathrm{OD})$, where $\rho$ is the density, $\kappa$ is the opacity, and $\delta$ OD is optical depth modulation. Figure 3(a) shows the $\lambda=$ $30 \mu \mathrm{m}$ side-by-side with $\lambda=10 \mu \mathrm{m}$ perturbation result and Figure 3(b) shows the $\lambda=50$ $\mu \mathrm{m}$ side-by-side with $\lambda=20 \mu \mathrm{m}$ perturbation result. 
(a) $30 \mu \mathrm{m} 10 \mu \mathrm{m}$

(b)
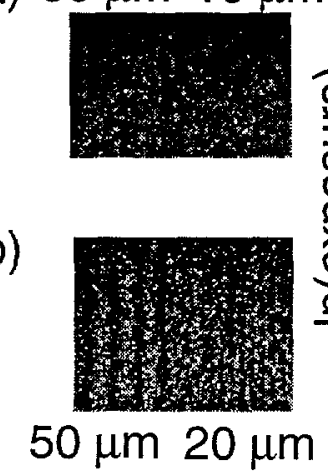

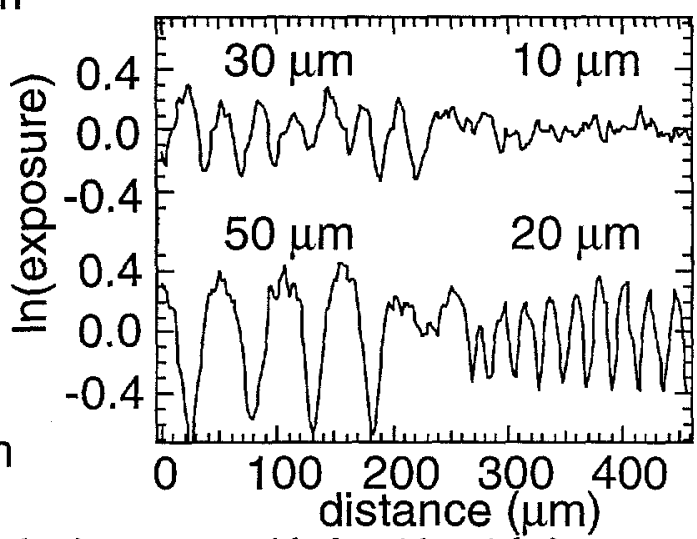

FIG. 3. (a) Radiographic image of the $\lambda=30 \mu \mathrm{m}$ side-by-side with $\lambda=10 \mu \mathrm{m}$ perturbation at $\mathrm{t}=4 \mathrm{~ns}$ along with the corresponding averaged lineout in $\ln$ (exposure). (b) Radiographic image of the $\lambda=20 \mu \mathrm{m}$ side-by-side with $\lambda=50 \mu \mathrm{m}$ perturbation at $\mathrm{t}=4 \mathrm{~ns}$ along with the corresponding averaged lineout in $\ln ($ exposure).

In order to extract the amplitude as a function of time from the image data, a region of each image was selected over which the backlighter intensity was approximately constant. The image intensity was converted to $\ln$ (exposure) using a calibrated transmission wedge for that piece of film. Optical depth as a function of position across the target was then extracted by averaging over the length of the perturbations. The lineouts in $\ln$ (exposure) were Fourier analyzed and the resulting mode amplitude was plotted as a function of the time for each image. These results were compared to two-dimensional simulations which were post-processed to produce amplitude in optical depth and convolved with the measured instrument response function. Figure 4 shows the comparison between the measured and simulated perturbation amplitudes at $10,12,16,20,30,50$, and $70 \mu \mathrm{m}$. No observable growth at $\lambda=10$ or $12 \mu \mathrm{m}$ is detected throughout the duration of the experimental measurements. Data taken on separate experiments are shown with different symbols. Reasonable agreeement is observed between the data and simulations as long as the measured drive conditions for each experiment are used to drive the corresponding simulation. 


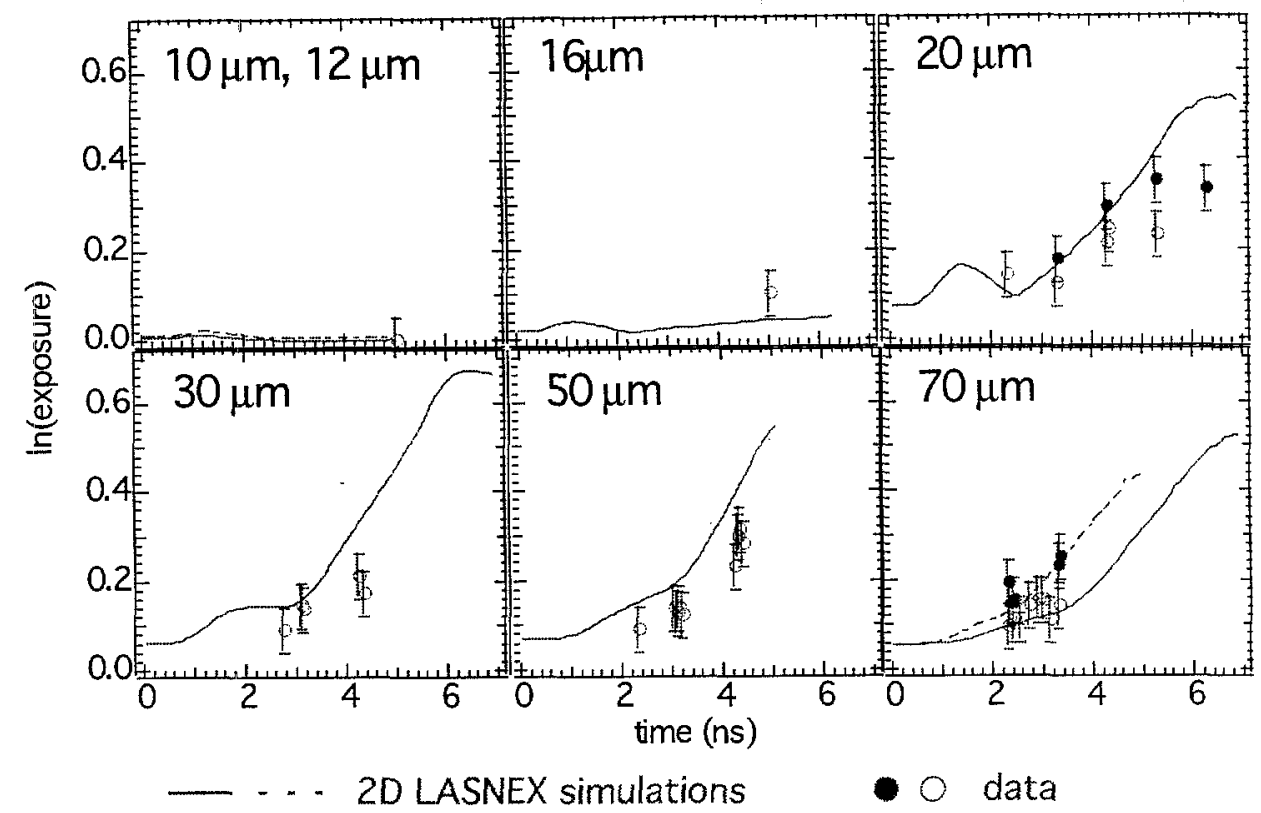

FIG. 4. Comparison between simulated and measured values of the optical depth modulation in $\ln$ (exposure) for the $10,12,16,20,30,50$, and $70 \mu \mathrm{m}$ wavelengths as a function of time.

Particularly at short wavelengths, there is a noticeable oscillation in the perturbation amplitude in both the data and simulations just prior to shock breakout time. Since the acceleration of the foil and corresponding Rayleigh-Taylor growth do not commence until the shock initially launched by the $\mathrm{x}$-ray drive has broken out of the rear surface of the target, it was suspected that this effect was correlated to the rippled shock moving through the thin foil. As the perturbed shock wave traverses the target it will oscillate, which in itself will not change the observed optical depth measurement. However, if material also flows laterally due to this oscillation this may increase or decrease the observed modulation. Since the oscillation of the rippled shock is expected to damp out over a distance roughly equal to the wavelength of the perturbation, this effect would be strongest at wavelengths close to the target thickness, $25 \mu \mathrm{m}$, as observed in the simulations and data. Direct comparison of the RT perturbation growth data to analytic models is difficult as they typically assume an isotropic, adiabatic compression and neglect the effect of shock waves. Since growth rates are extremely difficult to extract from the data due to the short duration of the acceleration phase of the experiment, the measured growth factor $\left.\mathrm{GF}=\eta(\mathrm{t}) / \eta_{o}\right)$ is compared to the 
simulated GF in Figure 5.

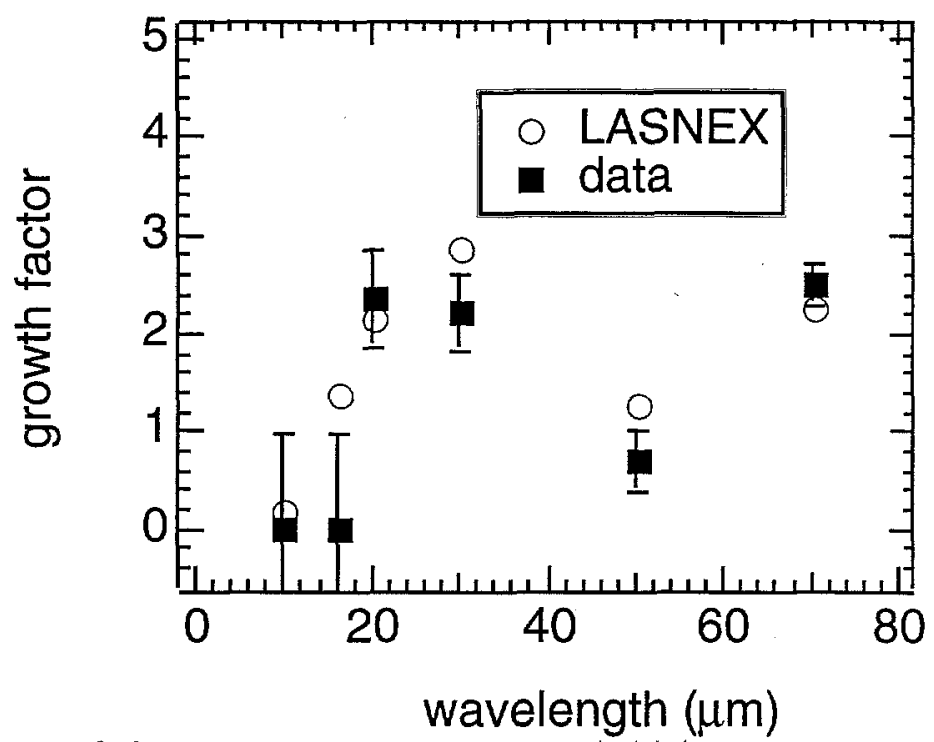

FIG. 5. Comparison of the measured growth factor $\left(\eta(\mathrm{t}) / \eta_{o}\right.$, open triangles) to the simulated growth factor (solid diamonds).

This early-time comparison shows reasonable agreement between the measured and simulated growth factors, except in the region of the roll-over of the growth. The roll-over occurs near wavelengths $\sim 20 \mu \mathrm{m}$ where the oscillation of the rippled shock should have a large effect. Additionally, due to the instrument response in this wavelength region rather large growth factors are required before the modulation will be seen in the data. The simulated growth factors at 3.2 ns are not large enough that we would expect any observable modulation growth in the data. The model of Betti et al. shows poor agreement with the experimental result due in large part to the considerable impact of the rippled shock transit on the data. Therefore, to enable more meaningful comparison with the analytical model a series of numerical experiment were undertaken that sought to remove or minimize the influence of the shock transit.

The calculation proceeded with no modulation at the ablation front until shortly after shock breakout for an unperturbed foil at $t=2.6 \mathrm{~ns}$ for a typical $\mathrm{X}$-ray drive used in the experiment. A small amplitude density perturbation (equivalent to an $\approx 0.001 \mu \mathrm{m}$ spatial perturbation) was then superimposed at the ablation front (taken to be the half-maximum 
density point). Calculations were carried out for each of the wavelengths used in the experiment to construct a dispersion curve for the instability and a respresentative pair of results is shown in Fig. 6.

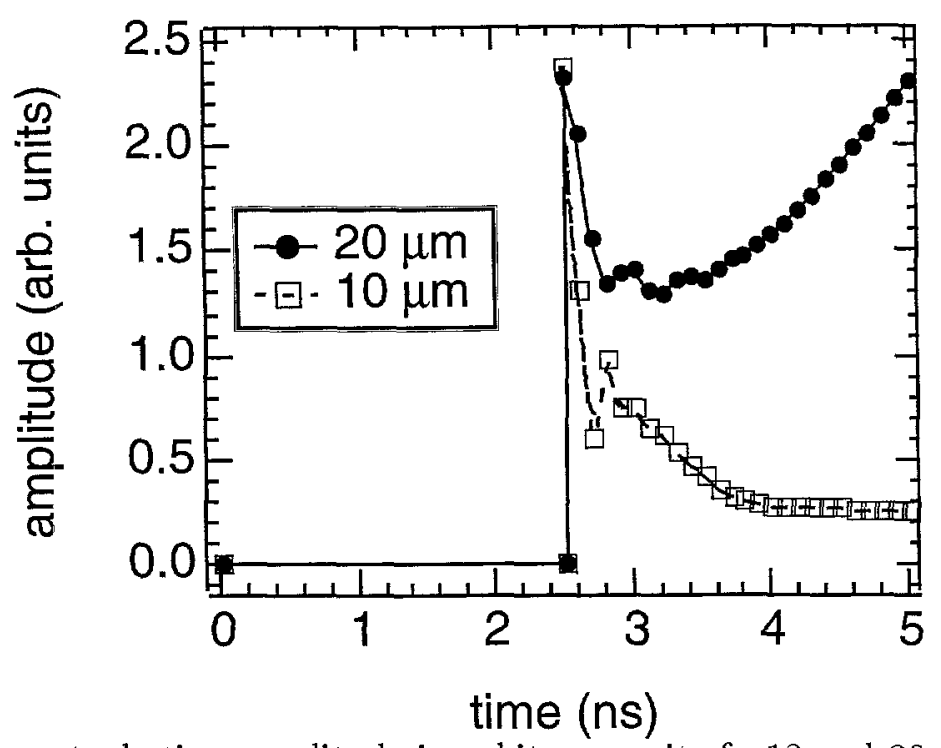

FIG. 6. Simulted perturbation amplitude in arbitrary units for 12 and $20 \mu \mathrm{m}$ wavelengths as a function of time for the numerical experiment simulation to remove the effect of the rippled shock transit.

Although this approach eliminates complicating growth during the shock transit phase, it is not without its own difficulties. Most notable is that the perturbation thus introduced is not in general an eigenmode of the system. As a result small oscillations are observed in the growth rates before they settle into smooth functions. However, by keeping the initial amplitude small the effect on the growth rates subsequent to the short oscillatory period are expected to be small.

The results of these simulations at $4 \mathrm{~ns}$, near the end of the acceleration phase, were used to construct a dispersion curve that was then compared to the analytic model of $R$. Betti and colleagues. [11] A one-dimensional LASNEX simulation of the foil motion for a characteristic $\mathrm{x}$-ray drive is used to generate pressure and density profiles at the ablation front as a function of time. The model then uses fits to these density and pressure profiles at each time step generated via the expressions 


$$
\begin{aligned}
& \frac{d \xi}{d y}=\frac{-\xi^{\nu+1}(1-\xi)}{\mathrm{L}_{\mathrm{o}}} \\
& \frac{\mathrm{P}_{\mathrm{a}} / \rho_{\mathrm{a}}}{\mathrm{V}_{\mathrm{a}}{ }^{2}} \frac{d \Pi}{d \mathrm{y}}=\xi^{-2} \frac{d \xi}{d y}+\frac{\xi}{\mathrm{L}_{\mathrm{o}} \mathrm{F}_{\mathbf{r}}}
\end{aligned}
$$

where $\nu$ is the power index for thermal conduction, $L_{0}$ is the characteristic spatial scale for thermal conductivity, and $\mathrm{F}_{\mathrm{r}}=\mathrm{V}_{\mathrm{a}}^{2} / \mathrm{gL}_{0}$ is the Froude number. In these equations the notation of Betti is used, in which $\xi$ and $\Pi$ are the density and pressure of the flow normalized to the peak density, $\rho_{\mathrm{a}}$, and pressure at peak density, $\mathrm{P}_{\mathrm{a}}$, respectively, and $\mathrm{y}$ is the spatial coordinate increasing in the direction of the flow. This model characterizes the hydrodynamic evolution primarily by the Froude number. Here the experimental package is characterized by a low Froude number and the growth rate of short wavelength modes is predicted to be reduced by a combination of transverse thermal conduction, the finite density gradient scale length and mass flow across the ablation front.

Figure 7 shows a representative set of equilibrium profiles generated by the onedimensional LASNEX simulation at 4 ns and the corresponding fit calculated with the model.

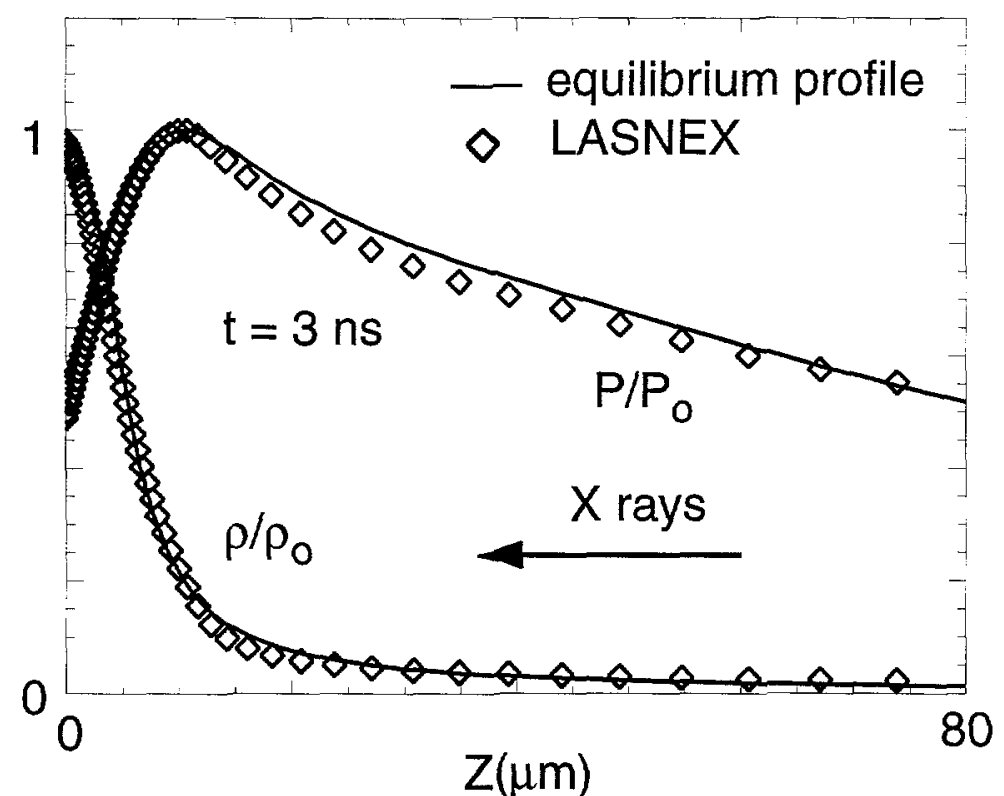

FIG. 7. One-dimensional simulated pressure and density profiles (solid lines) compared to the fits generated by the Betti model prescription (dashed lines) at $t=3 \mathrm{~ns}$.

The model was applied by choosing the set of parameters that provided the best fit to the 
density and pressure profiles predicted by a one-dimensional LASNEX simulation of the foil motion which used the same $\mathrm{X}$-ray drive as $2 \mathrm{D}$ instability calculations. The values extracted from these fits are then used to calculate the growth rate of a perturbation as a function of time. The values of $\nu, \mathrm{L}_{o}$, and $\mathrm{F}_{\mathrm{r}}$ increase slowly in time with typical values of $\nu \approx 1, \mathrm{~L}_{o} \approx 1.2$ corresponding to a minimum density gradient scale length of $\sim 5 \mu \mathrm{m}$ ), and $\mathrm{F}_{\mathrm{r}} \approx 0.2$. The ablation velocity remains between $1.3 \leq \mathrm{V}_{\mathrm{a}} \leq 1.8 \mu \mathrm{m} / \mathrm{ns}$ throughout the simulation and thus the acceleration decreases slowly from approximately $15 \mu \mathrm{m} / \mathrm{ns}^{2}$ at 3 $\mathrm{ns}$ to about half this value by $\sim 4.5 \mathrm{~ns}$. Figure 8 shows the growth rate as a function of wavelength, or dispersion curve, from the numerical experiment compared to the model of Betti and colleagues.

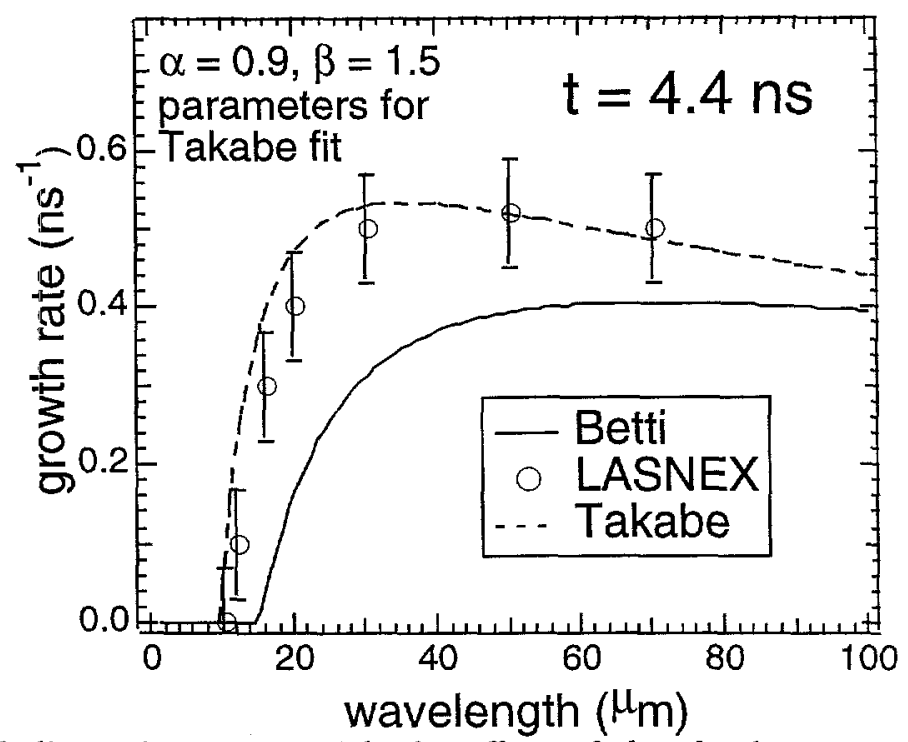

FIG. 8. Simulated dispersion curve with the effect of the shock transit phase removed (dots) and corresponding prediction from the model of Betti et al. (solid line) and the modified Takabe analysis (dashed line).

In this example, where the conditions of the simulation are better matched to the underlying assumptions of the analytic model, reasonably good agreement is observed between the model predictions and simulation. Note, the model has no free parrameters, other than the fitting to the pressure and density profiles to fix $\nu, \mathrm{L}_{0}$, and $\mathrm{F}_{\mathbf{r}}$.

These results were used to set the constants in a Takabe type growth rate formula 


$$
\gamma=\alpha \sqrt{\frac{\mathrm{Akg}}{(1+\mathrm{kL})}}-\beta \mathrm{kV}_{\mathrm{a}}
$$

where $\mathrm{k}$ is the wavenumber, $\mathrm{g}$ is the interface acceleration, $\mathrm{L}$ is the density gradient scale length defined as $\mathrm{L}_{o}(\nu+1)^{\nu+1} / \nu^{\nu}, \mathrm{V}_{\mathrm{a}}$ is the ablation velocity as described previously. It was found that setting $\alpha=0.92$, and $\beta=1.5$ results in excellent agreement for this case as expected for our application of the Takabe formula with $\beta$ used as a fitting parameter.

\section{CONCLUSIONS}

We have measured the Rayleigh-Taylor (RT) dispersion curve for a radiatively-driven sample in a series of experiments on the Nova laser facility. Planar aluminum foils were ablatively-accelerated and the subsequent perturbation growth was diagnosed via $\mathrm{x}$-ray radiography. These measurements unambiguously map out the linear regime dispersion curve, including the observation of stabilization at short wavelengths. The data are compared favorably to two-dimensional simulations. Due to the influence of the rippled shock transit phase of the experiment, direct comparison to the ablation front RT theory of R. Betti was difficult. Instead, a numerical "experiment" was constructed that minimized the influence of the shock and this was compared to the Betti model showing quite good agreement.

\section{ACKNOWLEDGMENTS}

The authors wish to acknowledge many fruitful discussions with T. S. Perry, the target fabrication efforts of H. Louis, T. Demiris, and R. Wallace, and the expert support of the Nova operations staff. This work was performed under the auspices of the U. S. Department of Energy by the Lawrence Livermore National Laboratory under Contract No. W-7405ENG-48. 


\section{REFERENCES}

[1] S. Chandrasekhar, Hydrodynamic and Hydromagnetic Stability, (Oxford University Press, London, 1968).

[2] D. Ryutov et al., Bull. Am. Phys. Soc. 1998.

[3] J. D. Lindl and W. C. Mead, Phys. Rev. Lett. 34, 1273 (1975); S. W. Haan, Phys. Rev. A 39, 5812 (1989); C. P. Verdon et al., Phys. Fluids 25, 1653 (1982); S. E. Bodner, Phys. Rev. Lett. 33, 761 (1974);J. D. Lindl, Phys. Plasmas 2, 3933 (1995).

[4] H. Takabe et al., Phys. Fluids 26, 2299 (1983); M. Tabak et al., Phys. Fluids B 2, 1007 (1990).

[5] M. M. Marinak, Phys. Plasmas 3, 2070 (1996).

[6] H. Azechi et al., Phys. Plasmas 4, 4079 (1997); S. G. Glendinning et al., Phys. Rev. Lett. 78, 3318 (1997); C. Pawley et al., Phys. Plasmas 6, 565 (1999); J. Knauer et al., Phys. Plasmas 7, 343 (2000).

[7] S. V. Weber et al., Phys. Plasmas 1, 3652 (1994).

[8] B. A. Remington et al., Phys. Fluids B 4, 967 (1992); B. A. Remington et al., Phys. Plasmas 2, 241 (1995).

[9] K. O. Mikaelian, UCID-19895 (1983); H. J. Kull, Phys. Rep. 206, 197 (1991) and references therein; R. Betti et al., Phys. Plasmas 2, 3844 (1995); M. Emery et al., Phys. Rev. Lett. 57, 703 (1986); A. Caruso and V. A. Pais, Laser and Part. Beams 12, 343 (1994).

[10] J. Grun et al., Phys. Rev. Lett. 58, 2672 (1987); M. Desselberger et al., Phys. Fluids B 5, 896 (1993); S. G. Glendinning et al., Phys. Rev. Lett. 69, 1201 (1992).

[11] R. Betti, R. L. McCrory, and C. P. Verdon, Phys. Rev. Lett. 7, 3131 (1993); R. Betti et al., Phys. Rev. E 50, 3968 (1994); R. Betti et al. Phys. Plasmas 2, 3844 (1995); R. 
Betti et al., Phys. Plasmas 3, 2122 (1996); V. Goncharov et al., Phys. Plasmas 3, 1402 (1996); R. Betti et al., Phys. Plasmas 5, 1446 (1998).

[12] K. S. Budil et al., Rev. Sci. Instrum. 67, 485 (1996).

[13] G. B. Zimmerman and W. L. Kruer, Comments Plasma Phys. Contol. Fusion 2, 51 $(1975)$. 